\title{
Tendências no Uso de Inteligência Artificial e sua Influência na Requalificação da Força de Trabalho no Setor Público
}

\author{
Trends in the Use of Artificial Intelligence and its Influence on the \\ Requalification of the Work Force in the Public Sector
}

\author{
Washington Fernando da Silva ${ }^{1}$ \\ Fernando Selleri Silva ${ }^{2}$ \\ Olivan da Silva Rabêlo \\ ${ }^{1}$ Universidade Federal de Mato Grosso, Cuiabá, MT, Brasil \\ ${ }^{2}$ Universidade do Estado de Mato Grosso, Barra do Bugres, MT, Brasil
}

\begin{abstract}
Resumo
Este estudo pretende identificar as tendências acadêmicas e tecnológicas, a partir de levantamento bibliográfico e patentário, sobre o uso da Inteligência Artificial (IA) no setor público e como esse uso poderá complementar, requalificar ou substituir a força de trabalho humana nos governos. Constatou-se que o uso de IA é crescente no setor público e que em alguns tipos de trabalho as tecnologias cognitivas poderão até substituir os servidores. O maior reflexo da IA no setor público será a complementação e a requalificação da força de trabalho, já que o seu uso pode resultar na liberação de até $50 \%$ do tempo dos servidores públicos, liberando-os para desenvolver habilidades nas quais as máquinas não superaram o trabalho humano. Os resultados apontam que as tecnologias de IA mudarão o funcionamento do governo e afetarão estruturalmente o planejamento, a estratégia e a gestão de recursos humanos, que deverá considerar o aumento da interação dos humanos com a tecnologia.
\end{abstract}

Palavras-chave: Inteligência Artificial. IA no Setor Público. IA na Gestão Pública. Força de Trabalho.

\begin{abstract}
Knowing the academic and technological trends, from a bibliographic and patent survey, on the use of $\mathrm{AI}$ in the public sector and how this use can complement, requalify or replace the human workforce in governments, this study found that the use of $\mathrm{AI}$ is growing in the public sector and that in some types of work cognitive technologies can even replace servers. The greatest reflection of $\mathrm{AI}$ in the public sector will be the complementation and requalification of the workforce, since its use can result in the liberation of up to $50 \%$ of public servants' time, freeing them to develop skills in which machines have not surpassed the human. The results indicate that AI technologies will change the functioning of government and will affect structurally, planning, strategy and human resource management, which will seek to evolve from the current focus on human talent to consider its interaction with technology.
\end{abstract}

Keywords: Artificial Intelligence. AI in the Public Department. AI in Public Management. Workforce.

Área Tecnológica: Administração. Inovação Tecnológica. Gestão Pública. Automação do Trabalho. 


\section{Introdução}

O século XX foi marcado por um processo de transformações políticas, sociais e econômicas que impactaram na reorganização do papel do Estado e da administração pública. Isso vem exigindo mudanças no modo de atender às demandas de cunho social, econômicas $e$ de infraestrutura, bem como requer o aperfeiçoamento e a criação de inovações nas funções estatais, nas estratégias de atuação governamental no mercado e na provisão de serviços públicos (CAVALCANTE; CAMÕES, 2017). A esse cenário soma-se a crescente demanda social por melhores serviços públicos, maior participação social e redução de custos no setor público (MEHR, 2017). Tal contexto justifica a importância de estudos e de práticas de inovação tecnológica e a adoção de tecnologias no setor público que permitam a maior eficiência e qualidade (CAVALCANTE; CAMÕES, 2017).

Entre as tecnologias emergentes, a IA se destaca como um campo de pesquisa interdisciplinar que recentemente ganhou importância especial na sociedade, na economia e no setor público, abrindo uma variedade de novas oportunidades, mas, também, de desafios (BOYD; WILSON, 2017). Rápidos avanços em automação e IA podem gerar um impacto significativo na maneira como se trabalha e na produtividade (BASTRA; QUEIROLO; SANTHANAM, 2018), haja vista que a IA pode complementar, ou até mesmo substituir, habilidades e tarefas da força de trabalho humana existente, gerando eficiência de custos e economia aos setores público e privado (WIRTZ; WEYERER; GEYER, 2019).

Nesse sentido, aplicações de IA estão sendo desenvolvidas em ritmo acelerado em universidades e por empresas de tecnologia como Apple, Facebook, Google, IBM e Microsoft, as quais investem fortemente para explorar aplicações de IA que consideram críticas para seus futuros (STONE et al., 2016). O emprego da IA já está mudando o cotidiano, transformando a estrutura de setores, como saúde e segurança, e contribuindo para o aumento da produtividade humana. Por outro lado, há discussões relevantes e, ainda pouco debatidas no campo científico, sobre o impacto dessas tecnologias na sociedade, na ética, na geração de emprego e na requalificação da força de trabalho (BORRY; GETHA-TAYLOR, 2018).

Torna-se relevante, portanto, a pesquisa sobre o uso da IA no setor público, no sentido de antecipar os impactos da automação na força de trabalho no setor governamental, delineando-se a questão de pesquisa deste trabalho da seguinte forma: Quais as tendências acadêmicas e tecnológicas sobre o uso da IA no setor público e suas implicações? Nesse contexto, o objetivo deste artigo é identificar tendências acadêmicas e tecnológicas sobre o uso da IA no setor público e como esse uso poderá complementar, requalificar ou substituir a força de trabalho humana nos governos, tencionando gerar produtividade, eficiência e efetividade para fazer frente às demandas de serviços de qualidade e, ao mesmo tempo, de ajuste fiscal nos múltiplos poderes do setor público.

Além de analisar quais os principais desafios e influências desse fenômeno, o artigo abrange a apresentação de referencial teórico, de estudo prospectivo baseado em documentos patentários e de discussões sobre previsões da automação com uso de IA no setor público. 


\section{Revisão da Literatura: a Inteligência Artificial (IA) e o futuro}

Embora o termo IA tenha sido explorado ao longo de décadas, ainda não existe uma definição consensual e globalmente aceita, dificultando seu estudo e entendimento (BORRY; GETHA-TAYLOR, 2018). A fim de se obter melhor compreensão do conceito de IA, pretende-se primeiro identificar o conceito de inteligência para depois aplicá-lo ao contexto de máquinas e sistemas.

Para Legg e Hutter (2007, p. 9), "A inteligência mede a habilidade de um agente em atingir objetivos em uma ampla gama de ambientes". Os autores propõem um conceito integrado de inteligência, descrevendo-a como a capacidade de interagir, aprender, adotar e de recorrer a informações a partir de experiências, bem como de lidar com incertezas. A partir do conceito de Legg e Hutter (2007), entende-se que o termo "artificial" significa uma réplica produzida por humanos, ou seja, a IA caracteriza-se como atividade dedicada a tornar máquinas e sistemas inteligentes (NILSSON, 2010).

Na dimensão da ciência, a IA é considerada um campo de pesquisa multidisciplinar (BOYD; WILSON, 2017) que visa a estudar como computadores podem fazer as tarefas, que, no momento, as pessoas ainda fazem melhor (RICH; KNIGHT; NAIR, 2009), pressupondo que programas computacionais são capazes de aprender, adaptar, ser criativos e de resolver problemas (ROSA; FEYEREISL; COLLECTIVE, 2016) e, por isso, podem vir a fazer essas atividades quase com a mesma habilidade, ou igualmente, ou até mesmo melhor do que um ser humano (THIERER; O'SULLIVAN; RUSSELL, 2017). Portanto, acredita-se que todos os aspectos da aprendizagem, ou qualquer outra característica da inteligência humana que pode, em princípio, ser precisamente descrita, podem ser simulados pelas máquinas (MCCARTHY et al., 2006).

A Inteligência Artificial pode ser dividida em três categorias principais: IA limitada (IAL), IA geral (IAG) e super IA (SIA) (WIRTZ; WEYERER; GEYER, 2019). A IAL geralmente é capaz de resolver um problema específico e precisa ser programada por humanos (ROSA; FEYEREISL; COLLECTIVE, 2016). Já a IAG é capaz de aprender por conta própria e transmitir suas experiências e habilidades para outras tarefas sem qualquer ajuda humana (ADAMS et al., 2012). Por fim, a SIA consiste no desenvolvimento de software mais avançado que a mente humana. Todavia, até este momento, tal software não existe, embora os cientistas acreditem que o SIA será uma evolução automática a partir da IAG (DELOITTE, 2018).

\subsection{A Inteligência Artificial no Setor Público}

A IA vem conquistando relevância especial na sociedade e no mercado, possibilitando inúmeras mudanças e novas oportunidades, inclusive para o setor público (MEHR, 2017). Embora haja pouco conhecimento sobre os tipos e potenciais de aplicações de IA para o setor público, as oportunidades de uso da IA nesse setor são imediatas e óbvias, especialmente para reduzir trabalhos administrativos, ajudar a resolver problemas de alocação de recursos e assumir tarefas significativamente complexas (MEHR, 2017). Tanto que o governo Chinês, por meio do Conselho de Estado da República Popular da China, emitiu uma diretriz com previsão de investimento de US $\$ 147,8$ bilhões para se tornar um desenvolvedor mundial de inovações em IA até 2030 ("China Issues Guideline On Artificial Intelligence Development", 2017). A China está rapida- 
mente lançando as bases para se tornar a líder da IA no mundo (FUTURE TODAY INSTITUTE, 2019). Os Estados Unidos vêm promovendo pesquisas e respectivos programas de educação em IA. Em 2016, gastaram aproximadamente US \$ 1,2 bilhão em pesquisa e desenvolvimento de tecnologias relacionadas à IA (HOLDREN; SMITH, 2016). A União Europeia relatou um investimento orçado de até 700 milhões de euros em robótica e em parcerias público-privadas envolvendo IA para o período de 2014 a 2020 (ANSIP, 2017).

Recentemente, Wirtz, Weyerer e Geyer (2019) fizeram uma abordagem conceitual que analisou e compilou percepções relevantes da literatura científica para fornecer uma visão geral $e$ integrativa de aplicativos de IA no setor público, além de desafios relacionados. O estudo sugeriu 10 áreas de aplicação da IA e identificou quatro dimensões principais dos desafios da IA no setor público (WIRTZ; WEYERER; GEYER, 2019). As 10 áreas apontadas foram: software de gerenciamento de conhecimento baseado em IA; sistemas de automação de processos; agentes virtuais; análise preditiva e visualização de dados; análise de identidade; robótica cognitiva $e$ sistemas autônomos; sistemas de recomendação; assistentes digitais inteligentes; análise de fala; análise de segurança cognitiva; e inteligência de ameaças (WIRTZ; WEYERER; GEYER, 2019).

As quatro dimensões principais dos desafios da IA foram divididas em: i) Implantação da tecnologia de IA; ii) Lei e regulamentação; iii) Ética; e iv) Sociedade (WIRTZ; WEYERER; GEYER, 2019). Na abordagem, os pesquisadores trazem uma análise detalhada sobre cada uma dessas dimensões na perspectiva de compreender os principais obstáculos e pontos de atenção para uma utilização crescente e progressiva da IA no setor público. Mehr (2017) faz uma abordagem de vários tipos de aplicativos de IA e os usos atuais e futuros na prestação de serviços ao cidadão pelo governo. Segundo a pesquisa, os usos de IA no setor público podem ser divididos em cinco categorias: respostas a perguntas, preenchimento e pesquisa de documentos, roteamento de petições, tradução e elaboração de documentos (MEHR, 2017). A pesquisa também propõe seis tipos de problemas que oportunizam a aplicação de IA no setor público, compreendendo a alocação de recursos, conjuntos grandes de dados, escassez de especialistas, cenário previsível, procedimento e dados diversos (MEHR, 2017).

As aplicações de IA para implantação no setor público tendem ao crescimento, com perspectivas de relevantes benefícios, como ajudar a reduzir o tempo de processamento, minimizar a carga e melhorar o fluxo de trabalho, aumentar a eficiência e impulsionar o crescimento econômico (CHATFIELD; REDDICK, 2018). Entretanto, essa tendência traz consigo desafios significativos que podem ser considerados críticos para que a adoção da IA no contexto do governo seja bem-sucedida, como a necessidade de recursos financeiros, os bloqueios à criatividade humana $e$ à inovação no setor público, a desconfiança social e teses relacionadas às substituições da força de trabalho humana pelas máquinas, gerando desemprego em grande escala, questões de ordem ética, normativa e legal, bem como pouca confiança social nos governos (WIRTZ; WEYERER; GEYER, 2019; MEHR, 2017; BOYD; WILSON, 2017).

\subsection{Como a IA Poderá Influenciar na Requalificação da Força de Trabalho Humana no Setor Público?}

A notória tendência de envelhecimento da população mundial e as mudanças ecossistêmicas culturais, tecnológicas e econômicas já se apresentam por si só como desafios na requalificação e na reformulação da força de trabalho humana mundial, tanto no setor privado quanto no 
setor público. Com agravante, no setor público, que, em linhas gerais, não tem sido capaz de acompanhar o ritmo de transformações dos negócios privados (MEHR, 2017). Uma grande mudança pouco estudada é a crescente ênfase na automação e as maneiras pelas quais essa opção tecnológica mudará a função e o contexto do trabalho (BORRY; GETHA-TAYLOR, 2018).

Um relatório recente evidenciou que 65\% dos americanos esperam que, dentro de 50 anos, robôs e computadores façam grande parte do trabalho feito por humanos (SMITH, 2016). Porém, apenas $7 \%$ dos americanos que trabalham no governo, educação ou setores sem fins lucrativos esperam que os robôs e computadores definitivamente assumirão a maioria dos empregos humanos nos próximos 50 anos (SMITH, 2016).

Um estudo realizado em 2013, por pesquisadores da Universidade de Oxford, estimou que 47\% de 702 empregos nos Estados Unidos estão em risco de "automação" (FREY; OSBORNE, 2013). A pesquisa concluiu que quanto menor o nível educacional exigido e o salário pago, maior a probabilidade de a ocupação ser informatizada (FREY; OSBORNE, 2013). Com base no trabalho original de Frey e Osborne (2013), dados do Banco Mundial sugerem que os riscos são maiores em muitos outros países; entre os países que compõem a Organização para a Cooperação e Desenvolvimento Econômico (OCDE), em média, 57\% dos empregos são suscetíveis à automação. Esse número sobe para 69\% na Índia e 77\% na China (MANYIKA et al., 2017). Essa abordagem contribui substancialmente por um lado, mas por outro, deixa lacunas de análise na medida em que sua escolha metodológica não considera com precisão as situações em que o trabalho ganha apenas suporte de IA, sem substituir totalmente a força de trabalho humana.

Na mesma linha, um estudo mais recente, realizado pela $\mathrm{PwC}$, estima que cerca de $38 \%$ dos empregos dos EUA e mais de um terço em outros países, como Alemanha, estão ameaçados, podendo deixar de existir até 2030 (BROWN et al., 2018). Nessa pesquisa são apresentadas tendências de mudanças no modelo de trabalho, como a diminuição drástica de trabalhos permanentes e estáveis, chegando a apenas 9\%, assim como a mudança das competências profissionais com maior valor no futuro, incluindo: adaptabilidade, resolução de problemas, habilidade colaborativa, inteligência emocional, criatividade e inovação, habilidades de liderança, habilidades digitais, gestão de riscos, habilidades STEM (Science, Technology, Engineering, and Math) e habilidades empreendedoras (BROWN et al., 2018).

Em uma visão consensual, é possível prever que, em alguns casos, a IA poderá, ao contrário de diminuir empregos, levar ao aumento de novos, relacionados direta e indiretamente ao desenvolvimento, supervisão e trabalho conjunto do humano com a máquina, já que pesquisas têm apontado o funcionamento da IA em sua maior parte com a colaboração de humanos (BROWN et al., 2018; MANYIKA et al., 2017; THIERER; O'SULLIVAN; RUSSELL, 2017; MEHR, 2017), afinal, muitos empregos ameaçados de desaparecerem hoje foram no passado os "disruptores" da antiga indústria (THIERER; O'SULLIVAN; RUSSELL, 2017). É um ponto quase homogêneo também que a máquina deve ser útil ao homem e não o contrário, e, se necessário, o estado deverá intervir, posteriormente, na criação de políticas públicas e de regulamentações para corrigir hipotéticos e eventuais impactos negativos, como o desemprego em massa, por exemplo (BROWN et al., 2018; THIERER; O'SULLIVAN; RUSSELL, 2017; STONE et al., 2016; MEHR, 2017). 


\section{Metodologia}

Trata-se de uma pesquisa de natureza aplicada, com vistas a estudar tendências acadêmicas, bibliográficas e patentárias do uso da IA no setor público, além de estudar como poderá complementar, requalificar ou substituir a força de trabalho humana. Quanto aos objetivos, tipifica-se como pesquisa exploratória, cujo objetivo é obter mais informações sobre um determinado tema, dando a ele um novo enfoque, por meio de levantamento de estudos científicos relevantes e de levantamento prospectivo em base patentária. No que se refere ao método, caracteriza-se como uma pesquisa bibliográfica ao buscar as informações sobre o tema proposto em publicações científicas e técnicas em base internacional (MARCONI; LAKATOS, 2003).

A abordagem utilizada é a análise qualitativa, na medida em que explora, significa e contextualiza o fenômeno da IA no serviço público, sob a ótica dos impactos e das transformações exigidas na força de trabalho humana no âmbito do governo, não fundamentada em dados estatísticos, mas em aspectos subjetivos do fenômeno (MARTINS, 2000). Ressalta-se que a realização da pesquisa foi organizada em etapas.

Na primeira etapa, foram definidos a questão de pesquisa e o objetivo do trabalho, como descrito na seção Introdução. Na segunda etapa, foram definidos descritores, baseados em palavras-chave retiradas da questão de pesquisa e do objetivo do trabalho, juntamente com seus sinônimos e palavras relacionadas. Posteriormente, foi escolhida a base Scopus para pesquisa de publicações científicas em virtude de sua abrangência, confiabilidade e acessibilidade quanto ao tema de pesquisa. Para os acessos às bases de informações científicas, foi utilizado o Portal de Periódicos da Coordenação de Aperfeiçoamento de Pessoal de Nível Superior (CAPES), órgão vinculado ao Ministério da Educação, do Governo Federal, por meio do acesso Comunidade Acadêmica Federada (CAFE). Foram realizados experimentos utilizando-se elementos de filtragem booleanos, truncagens e abrangência, a fim de definir a melhor composição para o êxito da pesquisa. Por fim, foram feitas as buscas em dois conjuntos de configurações. O primeiro com o objetivo de encontrar publicações que tratem da IA no setor público e o segundo que abordasse a IA e os impactos na força de trabalho, conforme pode ser observado no Quadro 1.

Quadro 1 - Critérios utilizados nas buscas de publicações científicas na base Scopus

\begin{tabular}{|c|c|c|c|}
\hline \multicolumn{2}{|c|}{ BusCA 1: IA NO SETOR PÚBLICO } & \multicolumn{2}{|c|}{ BuSCA 2: IA SEUS IMPACTOS NA FORÇA DE TRABALHO } \\
\hline Descritores & $\begin{array}{l}\text { "artificial intelligence", "AI", } \\
\text { "public sector" e "government" }\end{array}$ & Descritores & $\begin{array}{l}\text { "artificial intelligence", } \\
\text { "AI" e "workforce" }\end{array}$ \\
\hline $\begin{array}{l}\text { Truncagem, } \\
\text { booleanos } e \\
\text { abrangência }\end{array}$ & $\begin{array}{l}\text { "artificial intelligence" OR } \\
\text { "AI" (palavras-chave) } \\
\text { AND "public sector" OR } \\
\text { "government" (Título, } \\
\text { resumo e palavras-chave) }\end{array}$ & $\begin{array}{l}\text { Truncagem, } \\
\text { booleanos e } \\
\text { abrangência }\end{array}$ & $\begin{array}{l}\text { "artificial intelligence" } \\
\text { OR "AI" (palavras-chave) } \\
\text { AND "workforce" (Título, } \\
\text { resumo e palavras-chave) }\end{array}$ \\
\hline Período & $2017-2019$ & Período & $2015-2019$ \\
\hline $\begin{array}{l}\text { Quantidade de } \\
\text { publicações } \\
\text { encontradas }\end{array}$ & 57 & $\begin{array}{l}\text { Quantidade de } \\
\text { publicações } \\
\text { encontradas }\end{array}$ & 100 \\
\hline $\begin{array}{c}\text { Tipos de } \\
\text { documentos }\end{array}$ & Artigos; Revisões & Tipos & $\begin{array}{l}\text { (todos) Revistas; anais de } \\
\text { conferências; séries de livros; } \\
\text { livros; publicações comerciais; } \\
\text { paper; carta; editorial; } \\
\text { notas; pesquisa curta }\end{array}$ \\
\hline
\end{tabular}

Fonte: Elaborado pelos autores deste artigo 
Considerando que o fenômeno estudado é de caráter inovador e tecnológico, foi delimitado um período de três anos para a busca 1 e de cinco anos para a busca 2 . Ao concluir as buscas, foram realizadas leituras dos títulos de todos os trabalhos e do resumo de alguns, cujo título não deixava claro ser um trabalho relevante ou não para a pesquisa, além de observada a quantidade de citações dos trabalhos. Do universo de 157 resultados, refinou-se a pesquisa para 26 trabalhos, os quais foram devidamente analisados. O principal motivo de exclusão de resultados foi o fato de os trabalhos abordarem IA e setor público de forma distinta e não em conjunto. Adicionalmente, a fim de ampliar a cobertura de trabalhos relevantes, observou-se nos trabalhos incluídos as referências com maior incidência de citações, que se constituem como balizadoras para o estudo do tema, algumas pesquisas científicas, como a pesquisa de Frey e Osborne (2013), e outras editoriais ou comerciais, como as pesquisas da McKinsey Global Instituto (MANYIKA et al., 2017) e da PwC (BROWN et al., 2018). Portanto, foram estudados artigos adicionais selecionados a partir das referências dessas publicações em uma abordagem de aprofundamento.

Os passos seguidos para elaboração do estudo prospectivo se iniciaram com a definição do objeto e do campo de busca, seguido da delimitação das palavras-chave, a classificação, a escolha da base de dados e a ferramenta de busca, conforme detalhamento no Quadro 2. Os descritores escolhidos são genéricos, dada a dificuldade de correlacionar as publicações patentárias ao tema de requalificação da força de trabalho, visto que, em sua maioria, as tecnologias não buscam primariamente esse objetivo. A requalificação da força de trabalho apresenta-se como uma consequência geral das tecnologias de IA.

Quadro 2 - Critérios utilizados nas buscas de documentos de patentes na base Orbit

\begin{tabular}{|c|c|}
\hline DESCRIÇÃO DOS CRITÉRIOS DE BUSCA & DESCRITOR \\
\hline Objeto de busca & $\begin{array}{l}\text { Depósitos de patentes em Inteligência } \\
\text { Artificial em todo o mundo. }\end{array}$ \\
\hline Campos de Busca & $\begin{array}{c}\text { Título } \\
\text { Resumo } \\
\text { Reivindicações } \\
\text { Descrição } \\
\text { Objeto da invenção } \\
\text { Vantagens ou desvantagens sobre o estado da técnica } \\
\text { Reivindicações independentes } \\
\text { Conceitos }\end{array}$ \\
\hline Palavras-chave e booleanos & $\begin{array}{c}\text { (IA OR Artificial Intelligence) } \\
\text { AND } \\
\text { ("Smart*" OR "Robo*") }\end{array}$ \\
\hline Classificação & Todas \\
\hline Periodicidade & Sem restrição \\
\hline Base de dados & Orbit: www.orbit.com \\
\hline
\end{tabular}

Fonte: Elaborado pelos autores deste artigo 


\section{Discussões e Análise dos Resultados: Estudo Prospectivo de Patentes relacionadas à Inteligência Artificial}

Foram encontradas 25.432 publicações de patentes relacionadas à Inteligência Artificial com presença das palavras-chave IA ou Artificial Intelligence e palavras cujo prefixo sejam "smart" ou "robô" (QUESTEL, 2019).

Ao realizar a análise sobre a tendência de investimento em tecnologia de IA, observa-se pelo Gráfico 1 a evolução das aplicações ao longo do tempo, indicando a dinâmica de inventividade da carteira estudada. Pode-se perceber uma carteira crescente e exponencial, o que geralmente é sintomático de uma forte tendência de investimento em P\&D na dimensão tecnológica analisada. Vale ressaltar que sempre haverá uma lacuna na informação de patentes atuais devido ao atraso de 18 meses entre a apresentação de um pedido e sua publicação (QUESTEL, 2019).

Gráfico 1 - Tendência de investimento em IA baseado em números de patentes

Investment trend

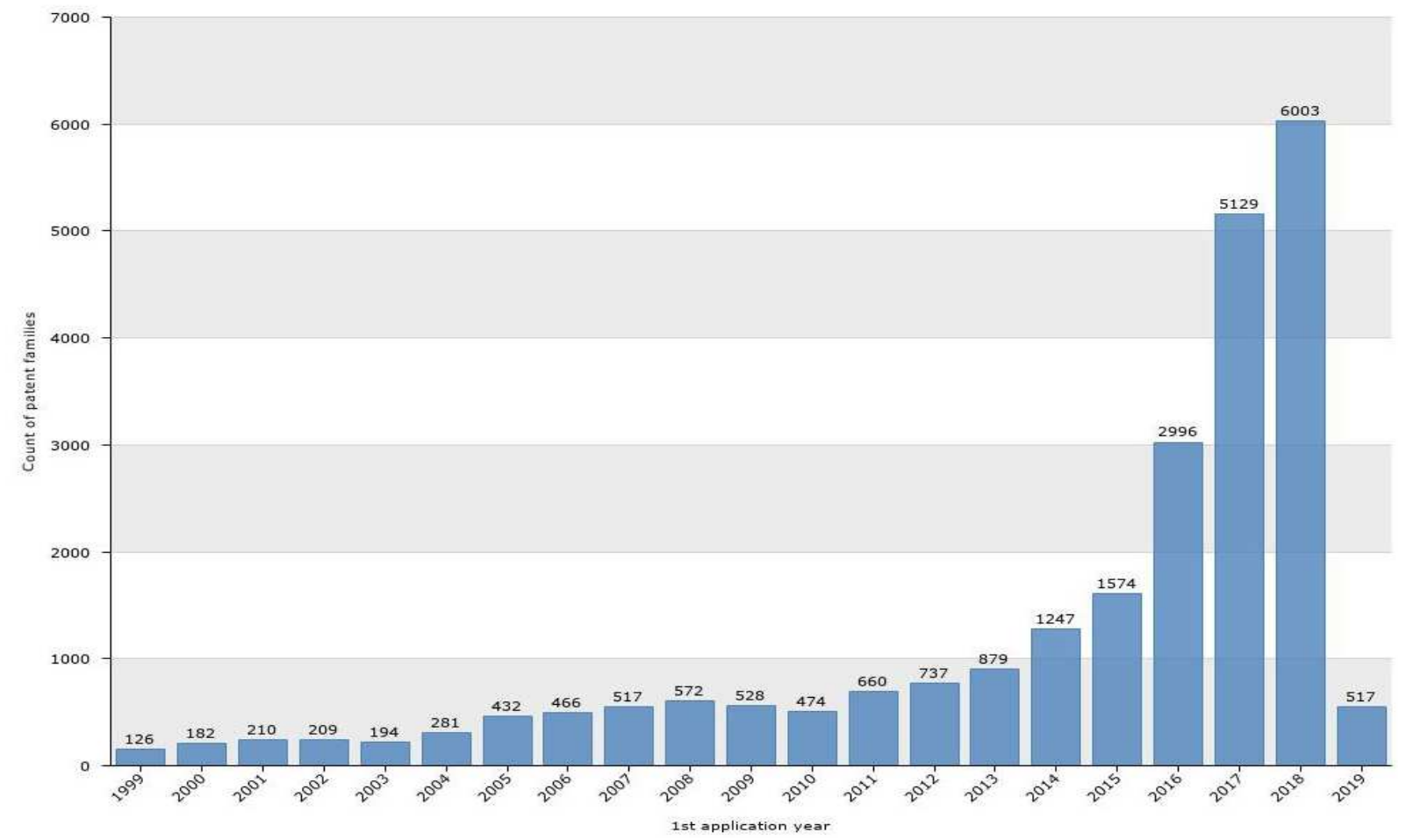

Fonte: Questel (2019)

O Gráfico 2 mostra o tamanho das carteiras dos requerentes no grupo de patentes analisado. Esses dados são um bom indicador do nível de inventividade dos atores ativos. Identificou-se que as 10 maiores organizações requerentes de patentes em IA são, respectivamente: Samsung, com 338 depósitos; Beijing Guangnian, com 217; State Grid, com 181; Microsoft, com 119; Beijing University, com 107; IBM, com 101; South China University, com 100; Google, com 88; LG Electronics, com 87; e Intel, com 84. Na China e nos Estados Unidos encontram-se mais de $50 \%$ das patentes protegidas vivas, sendo que $40,66 \%$ dos registros foram requeridos na China.

Esses números demonstram a estratégia de proteção dos requerentes e ajuda a identificar os principais mercados-alvo. Por meio da carteira de patentes analisada foi possível deduzir que o principal mercado do mundo é a China, conforme observa-se no Gráfico 3. 
Gráfico 2 - Principais requerentes de patentes relacionadas à Inteligência Artificial

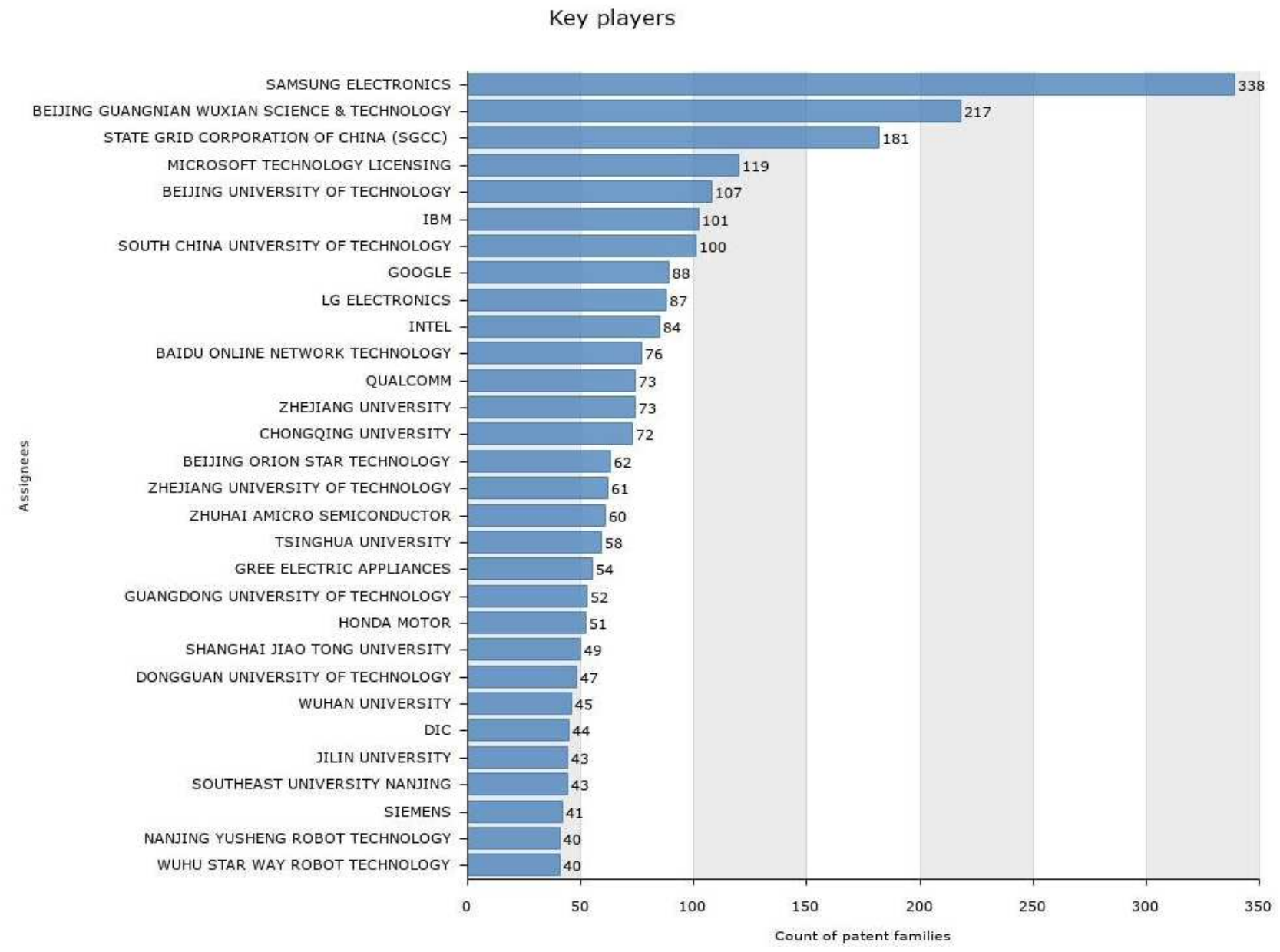

Fonte: Questel (2019)

Gráfico 3 - Proteção de patentes relacionadas à Inteligência Artificial por países

Protection country

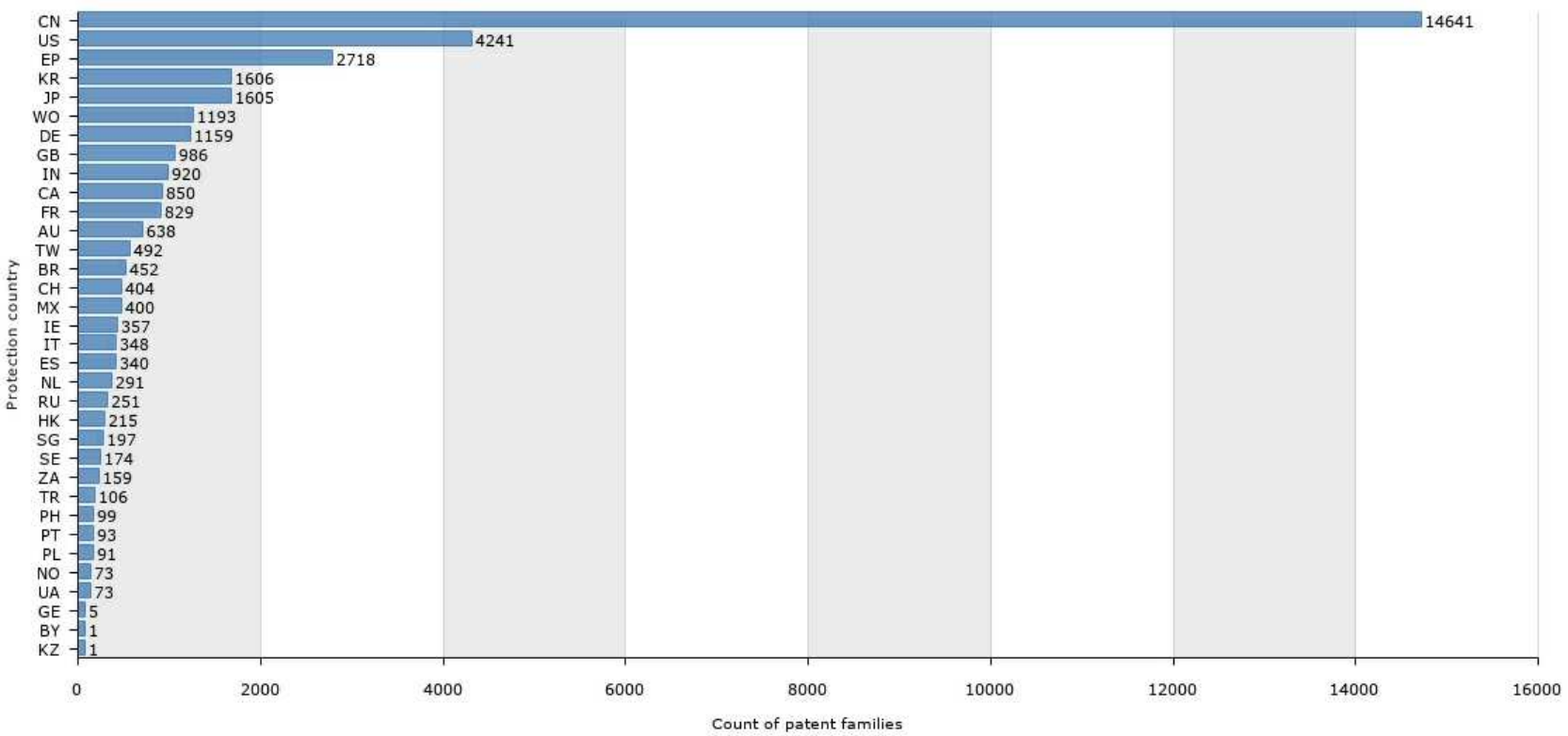

Fonte: Questel (2019) 
No Gráfico 4, é possível visualizar a distribuição das patentes analisadas por códigos de Classificação Internacional de Patentes (CIP). Os códigos CIP foram agrupados em 35 campos de tecnologia, que são representados no gráfico. É importante evidenciar que as categorizações pelo domínio da tecnologia são baseadas em agrupamentos de código CIP, assim, as patentes podem aparecer em diversas categorias diferentes. Ao analisar os dados obtidos na pesquisa, foi possível identificar que as principais categorias abarcadas pelos requerentes foram Computer Technology, com 6.570 patentes; handling, com 5.821; control, com 4.805; IT methods for management, com 2.412; measurement, com 2.267; digital communications, com 2.200; telecommunications, com 1.965; furniture games, com 1.614; transport, com 1.476; e medical technology, com 1.475.

Gráfico 4 - Patentes por Classificação Internacional de Patentes (CIP)

Patent families by Technology domain

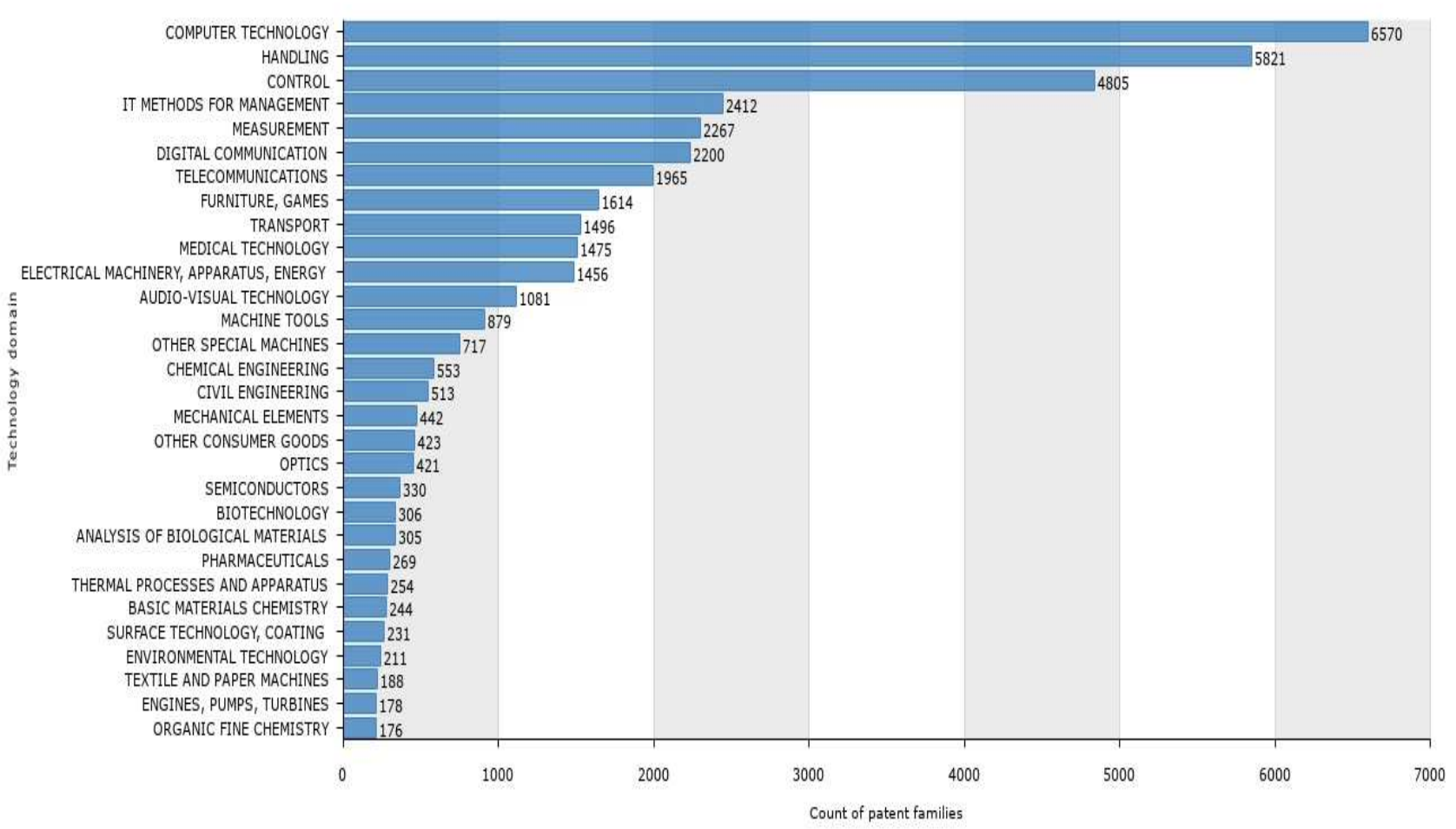

Fonte: Questel (2019)

Por fim, foram analisados os principais conceitos contidos na carteira de patentes de Inteligência Artificial selecionada. No Gráfico 5, é possível ilustrar os conceitos mais utilizados pelos requerentes. Essa análise é relevante, pois pode ser uma fonte de ideação para novos desenvolvimentos ou identificação de tecnologias protegidas em um novo campo.

Ao todo foram identificados 70 conceitos utilizados distribuídos em seis grandes categorias: Robo Body, Learning, Intelligent Robot, Voice Command, Robo Motion, Sweeping robot, obstacle avoidance, chat robô e hedge Equalization Economics - Cloud. 
Gráfico 5 - Conceitos contidos na carteira de patentes analisadas

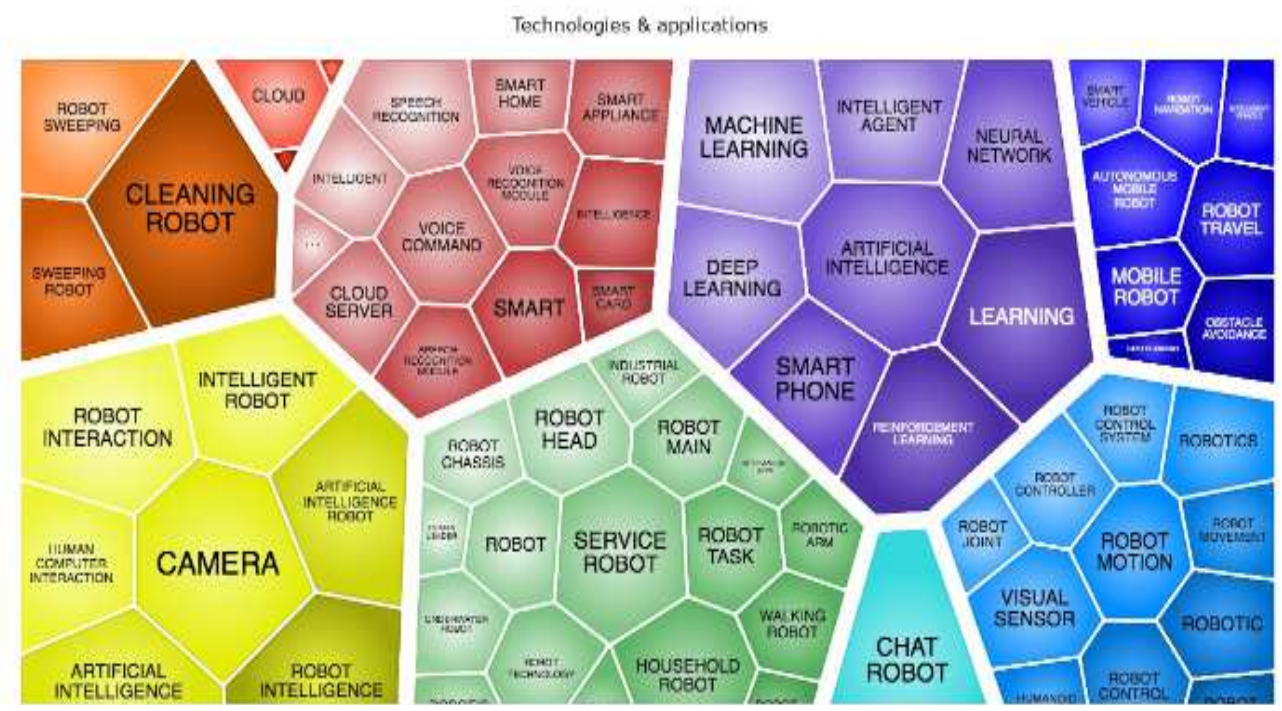

Fonte: Questel (2019)

Vale ressaltar que esta pesquisa não tem o objetivo de trazer referencial teórico para explicar tais conceitos, mas apenas sinalizá-los, a fim de despertar a atenção de futuras pesquisas para o devido aprofundamento no tema.

\section{Discussões Sobre Previsões da Automação com Uso de IA no Setor Público}

Embora a pesquisa empírica sobre IA no setor público ainda seja escassa (SUN; MEDAGLIA, 2019), a partir dos trabalhos encontrados, foi possível construir uma análise cuja síntese e discussões encontram-se a seguir.

Considerando que o progresso de trabalhos científicos publicados sobre esse tema está previsivelmente aumentando, pode-se auferir uma tendência de pesquisa sobre o assunto (REIS; SANTO; MELÃO, 2019). Similarmente aos depósitos de patentes, os artigos publicados sobre IA aumentaram exponencialmente nos últimos anos (REIS; SANTO; MELÃO, 2019). Apesar de as discussões e as evidências científicas de adoção da Inteligência Artificial no setor privado estarem mais fortalecidas e expressivas, o uso de tecnologias baseadas em IA já está presente no setor público, transformando a forma como os serviços do governo funcionam (REIS; SANTO; MELÃO, 2019).

Tecnologias de IA no setor público têm simplificado mecanismos burocráticos, reduzindo tempo de resposta, agilizando o processamento de dados, facilitando a tomada de decisão, promovendo formas inovadoras de comunicação entre o governo e os cidadãos, melhorando e até transformando completamente a prestação de serviços públicos (ANDROUTSOPOULOU et al., 2019; CENTRE FOR PUBLIC IMPACT, 2017; REIS; SANTO; MELÃO, 2019; WIRTZ; WEYERER; GEYER, 2019).

O estudo não exaustivo em publicações bibliográficas possibilitou constatar inúmeras alternativas e possibilidades de aplicações da IA no setor público. Por exemplo, na área da saúde pública, a IA tem auxiliado no tratamento e no monitoramento de pacientes, diagnósticos clí- 
nicos precisos, gestão de riscos de disseminação de doenças em ambientes externos e internos, predição de epidemias na sociedade e robótica na assistência de cirurgias (COLLIER et al., 2017; SUN; MEDAGLIA, 2019). As aplicações de IA são a base da chamada medicina personalizada e têm colaborado para diagnósticos, tratamentos e monitoramentos clínicos cada vez mais personalizados e precisos, na medida em que proporcionam maior exatidão nas decisões médicas. Como é o exemplo do uso do IBM Watson na área oncológica pelos governos chinês e americano, cujos resultados observados no combate ao câncer tornaram-se expressivamente superiores aos métodos tradicionais (CENTRE FOR PUBLIC IMPACT, 2017; SUN; MEDAGLIA, 2019).

Na educação, as influências da digitalização e a democratização do acesso à informação em plataformas on-line têm levado os estudiosos a preverem mudanças estruturais e possíveis rupturas no modelo educacional público. Escolas estão experimentando sistemas de ensino completamente diferentes, como é o caso de uma rede de nove escolas na Califórnia, em que os alunos passam um quarto do dia usando uma plataforma on-line sem a necessidade de um professor real e usam o tempo em sala de aula para fazer as atividades que, antes, eram a lição de casa. Essa inversão na sala de aula tende a se tornar uma realidade em todos os níveis de formação educacional devido à massiva oferta de conteúdos educacionais gratuitos on-line, como YouTube EDU, TED-Ed e Khan Academy (CENTRE FOR PUBLIC IMPACT, 2017; STONE et al., 2016).

Estudos apontam que as aplicações da IA juntamente com outras tecnologias emergentes poderão causar transformações sem precedentes na forma que o governo provê educação para os cidadãos, especialmente promovendo personalização em escala (STONE et al., 2016). Professores robôs inteligentes estão interagindo com os alunos e ensinando ciências, matemática, idiomas e outras disciplinas (STONE et al., 2016). Sistemas inteligentes estão permitindo aos professores aumentarem o tamanho de suas salas e desenvolverem abordagens de aprendizagem personalizadas a cada aluno. Estudiosos apontam um crescimento na oferta de ensino a distância e no uso de tecnologias de IA integrados ao modelo presencial face a face (STONE et al., 2016).

Na segurança pública, estudos apontam a aplicação da IA no policiamento preditivo, no combate ao crime e na gestão prisional (CENTRE FOR PUBLIC IMPACT, 2017; KOUZIOKAS, 2017; WIRTZ; WEYERER; GEYER, 2019). Os departamentos de polícia passaram a ter capacidade de prever a atividade criminosa, o Departamento de Polícia de Santa Cruz, na Califórnia, testou uma ferramenta de análise baseada em IA para prever pontos críticos de crime, contribuindo para uma redução de $27 \%$ dos crimes contra a propriedade (GOLDSMITH; CRAWFORD, 2014). Postes inteligentes com IA coletam e analisam dados em tempo real, podendo rastrear movimentos de pedestres, decidir quando apagar ou acender cada lâmpada, alertar os cidadãos sobre vagas de estacionamento livres, entre outras possibilidades (GOLDSMITH; CRAWFORD, 2014). O FBI criou a Unidade de Comparação e Avaliação de Análise Facial (FACE), que fornece suporte investigativo usando software de reconhecimento facial para comparar imagens faciais contidas em sistemas governamentais (por exemplo, fotografias do Departamento de Veículos Motorizados) com fotos de pessoas desaparecidas e de fugitivos (CENTRE FOR PUBLIC IMPACT, 2017).

Sistemas computacionais cognitivos também têm sido utilizados para gestão inteligente de tráfego urbano, suportam cenários complexos de tomada de decisão em tempo real devido à 
sua capacidade de trabalhar com grandes quantidades de dados e conectar pontos a uma taxa muito maior de eficácia e eficiência (DESOUZA, 2018). Na justiça, os sistemas inteligentes estão ajudando a reduzir o tempo e as inconsistências na tomada de decisão, algoritmos fornecem previsões precisas sobre risco e custo que um indivíduo pode representar à sociedade, baseadas em dados processuais (CENTRE FOR PUBLIC IMPACT, 2017).

A IA também vem sendo utilizada para formulação de políticas públicas, ajudando os governos a agregar e a analisar os interesses da população por meio de vários algoritmos que podem fornecer e processar informações de plataformas de mídia social para identificar problemas $e$ avaliar o sentimento público sobre uma determinada política pública (CENTRE FOR PUBLIC IMPACT, 2017; PATIL et al., 2018).

As possibilidades de uso da IA no setor público são variadas, interfaces de usuário de sistemas de software, interação humano-computador para tarefas repetitivas como entrada de dados, agentes virtuais (chatbots e avatares), análise preditiva com aprendizagem de máquina (machine learning) e visualização de dados, softwares combinados com big data de análise avançada de dados (DESOUZA, 2018; MEHR, 2017; STONE et al., 2016; WIRTZ; WEYERER; GEYER, 2019).

Embora sejam previstos muitos benefícios que a IA trará para o setor produtivo (FREY; OSBORNE, 2013) e governamental (MEHR, 2017), muitos relatórios apontam o risco de desemprego (BOYD; WILSON, 2017; BROWN et al., 2018; FREY; OSBORNE, 2013). Segundo essa linha, existe o risco de os sistemas atuais se tornarem injustos com a chegada da IA e acumularem um grande número de cidadãos economicamente sem valor e uma minoria de pessoas tecnologicamente alfabetizadas que adquirem riqueza e influência sem precedentes (BOYD; WILSON, 2017; BROWN et al., 2018; FREY; OSBORNE, 2013). Nesse cenário potencial, seria necessária uma nova e justa distribuição de renda, pois muitos trabalhadores começariam a sofrer sem culpa própria (BOYD; WILSON, 2017). Uma solução cogitada por vários autores para essa questão é uma renda básica universal financiada pela taxação de robôs que substituiriam os trabalhadores humanos (WELLER, 2017).

Por uma outra ótica, autores defendem que, assim como nas revoluções agrícolas e industriais do passado, surgiriam novos tipos de trabalho e haveria muito mais colaboração entre homem e máquina do que a substituição completa da mão de obra humana (MEHR, 2017). Nessa perspectiva de cenário, o governo deveria prover a requalificação da força de trabalho a fim de mitigar as lacunas de qualificação técnica em relação às novas demandas do mercado de trabalho. Essa perspectiva defende que as máquinas sempre servirão aos humanos e não o contrário (EGGERS; SCHATSKY; VIECHNICKI, 2017; MEHR, 2017; STONE et al., 2016).

$\mathrm{Na}$ dimensão ética, é possível citar o caso de preconceito indutivo no uso da IA para prever chances de reincidência de presidiários nos Estados Unidos (EGGERS; SCHATSKY; VIECHNICKI, 2017). Os algoritmos parecem ter absorvido o racismo em suas suposições programadas nele, pois réus erroneamente rotulados como de alto risco eram duas vezes mais propensos a serem negros, enquanto aqueles erroneamente rotulados como de baixo risco eram muito mais prováveis serem brancos (EGGERS; SCHATSKY; VIECHNICKI, 2017). As questões éticas envolvendo um equilíbrio entre privacidade e aquisição de dados, também, são consideradas desafios da IA para o setor público (EGGERS; SCHATSKY; VIECHNICKI, 2017).

Para adoção de IA no setor público, existem obstáculos de gestão e organizacionais que incluem desafios relacionados à estratégia, aos recursos humanos e às práticas de gerenciamento de cada organização pública na adoção da IA (SUN; MEDAGLIA, 2019). Compõem essa di- 
mensão a possível resistência organizacional ao compartilhamento de dados; a falta de pessoal interno qualificado apropriadamente (CENTRE FOR PUBLIC IMPACT, 2017); a falta de planos estratégicos para o desenvolvimento de IA (SUN; MEDAGLIA, 2019); e as possíveis resistências sindicais frente à ameaça de substituição da força de trabalho humana (ANDROUTSOPOULOU et al., 2019; MEHR, 2017).

Os desafios culturais se referem às resistências humanas para a inovação no setor público e devido à falta de confiança dos empregados públicos e dos cidadãos nas máquinas (ANDROUTSOPOULOU et al., 2019; CENTRE FOR PUBLIC IMPACT, 2017; SUN; MEDAGLIA, 2019). Por exemplo, um robô poderia, em teoria, ser capaz de substituir algumas das funções de uma enfermeira. Mas, por enquanto, a perspectiva de que isso possa realmente acontecer de uma maneira altamente visível poderia ser inaceitável para muitos pacientes que esperam e confiam no contato humano (MANYIKA et al., 2017).

Ameaças à segurança pública diante de empresas multinacionais que coletam dados sensíveis do governo e da sociedade, somadas à falta de regras de responsabilidade no uso da IA (SUN; MEDAGLIA, 2019) e de privacidade e segurança de dados (KANKANHALLI; CHARALABIDIS; MELLOULI, 2019), constituem-se como desafios políticos e legais da IA para o setor público. Os princípios gerais de direito existentes são para regular o comportamento dos seres humanos e não o das máquinas. Assim, a implantação adicional de sistemas de IA causará inevitavelmente o surgimento de novos conceitos e regulamentos legais que esclareçam e incentivem o compartilhamento de dados entre as partes interessadas, bem como regulará o uso dos dados públicos e as responsabilidades sobre as atividades dos sistemas computacionais e robôs inteligentes (MIKHAIL; ALEKSEI; EKATERINA, 2018).

\section{IA e seus Efeitos na Força de Trabalho Humana no Setor Público}

A IA apresenta aos governos novas opções sobre como realizar o trabalho, possibilitando substituir completamente o humano em alguns casos e, em outros, assumindo parcialmente as atividades realizadas pelos empregados públicos (FREY; OSBORNE, 2013; MANYIKA et al., 2017). A IA poderá proporcionar a cooperação e a interação entre pessoas e máquinas tornando possível aprimorar os resultados como um todo (EGGERS; SCHATSKY; VIECHNICKI, 2017). Há fortes preocupações sobre um possível desemprego em massa decorrente da automação por tecnologias de IA no setor privado (BHATTACHARYYA; NAIR, 2019; BROWN et al., 2018; FREY; OSBORNE, 2013; SMITH, 2016) que também preocupam o setor público (BORRY; GETHA-TAYLOR, 2018).

O grau de automação potencial para os diversos serviços e para os trabalhos públicos são diversos (MANYIKA et al., 2017). Os com maior probabilidade de serem substituídos envolvem atividades físicas e cognitivas previsíveis e padronizáveis. De forma geral, ocupações com salários mais altos e maiores requisitos de habilidade têm menor potencial de automação (BORRY; GETHA-TAYLOR, 2018; BROWN et al., 2018; MANYIKA et al., 2017; STONE et al., 2016). Estudos apontam que, com as tecnologias existentes, um hospital já poderia ter cerca de $70 \%$ da sua atividade automatizada e obteria um ganho de cerca de $30 \%$ na sua produtividade (CENTRE FOR PUBLIC IMPACT, 2017). A tecnologia geraria um aumento da produtividade 
de enfermeiros e médicos, a redução do tempo de espera do paciente e melhores resultados na área da saúde (MANYIKA et al., 2017).

Os estudos revelam que, no futuro, os espaços de trabalhos tendem a favorecer o trabalho colaborativo e em rede (BHATTACHARYYA; NAIR, 2019). As tecnologias permitirão mensurar produtividade dos trabalhadores, mesmo que estes trabalhem de suas casas ou em escritórios de uso compartilhado (coworking), tornando as jornadas e os locais de trabalho, em muitos casos, totalmente flexíveis e possibilitando o trabalho remoto (BHATTACHARYYA; NAIR, 2019). O contexto futuro tornará a liberdade de trabalho tão importante para os funcionários públicos quanto os incentivos financeiros (BROWN et al., 2018).

É provável que esse quadro influenciará grande parte das agências públicas do mundo a repensarem as estruturas das carreiras públicas vigentes e sua estratégia geral de recursos humanos (DESOUZA, 2018). É um desafio prever as habilidades que serão necessárias, mesmo daqui a cinco anos, portanto, o servidor público precisará se adaptar às mudanças organizacionais (DESOUZA, 2018) cada vez mais frequentes, além de estar disposto a adquirir novas habilidades e experiências ao longo de sua vida para tentar realizar novas tarefas e, até mesmo, para se requalificar em sua carreira.

\section{Considerações Finais}

Este trabalho buscou identificar tendências acadêmicas e tecnológicas sobre o uso da IA no setor público e suas implicações. Assim, observou-se que à medida que os processos são transformados pela automação de atividades individuais, as pessoas realizam atividades que são complementares ao trabalho que as máquinas fazem (MANYIKA et al., 2017). Nossa análise é que, mesmo em cenários de forte automação, os seres humanos ainda serão necessários na força de trabalho e os ganhos totais de produtividade que são estimados pelos estudos só acontecerão se as pessoas trabalharem lado a lado com as máquinas.

Constatou-se que o desenvolvimento e o uso de tecnologias de IA apresentam crescimento potencial, o mundo passa por uma nova era de automação, na qual robôs e sistemas computacionais executam uma série de atividades de trabalho físico de rotina de forma mais eficiente e barata que os humanos (por exemplo fazer julgamentos tácitos, capturar e reproduzir emoções e dirigir), assim esses robôs se tornarão cada vez mais capazes de realizar atividades que incluem capacidades cognitivas (BHATTACHARYYA; NAIR, 2019; BOYD; WILSON, 2017; BROWN et al., 2018; MANYIKA et al., 2017; STONE et al., 2016). O ritmo, a extensão da automação e, portanto, o seu impacto sobre a força de trabalho humana variam em diferentes atividades, ocupações e níveis salariais e de qualificação. Muitos trabalhadores continuarão trabalhando ao lado de máquinas, já que várias atividades são automatizadas apenas parcialmente (BORRY; GETHA-TAYLOR, 2018; BROWN et al., 2018; MANYIKA et al., 2017; STONE et al., 2016).

A automação de atividades pode permitir o crescimento da produtividade, as reduções de custos, maior qualidade, eficiência, eficácia e efetividade dos serviços e prover atendimento perpétuo digital 24 horas ao dia aos cidadãos (DESOUZA, 2018; FREY; OSBORNE, 2013; MANYIKA et al., 2017; MEHR, 2017). As tecnologias cognitivas provavelmente mudarão fundamentalmente o funcionamento do governo e afetarão o planejamento e a gestão dos recursos humanos que necessitará evoluir do atual foco no talento humano, para considerar a interação 
do humano e a tecnologia. Nossa análise é que a inteligência artificial e os sistemas cognitivos poderão resultar na substituição de alguns empregos públicos devido à automação, mas também aumentar a qualidade do trabalho que está sendo realizado e abrir novas oportunidades de trabalho. Transformar a força de trabalho do setor público não tem sido fácil para a maioria dos governos, mas ignorar a questão só complicará as coisas no futuro.

Os formuladores de políticas devem enfrentar escolhas sobre como aplicar essas tecnologias no setor público. Essas escolhas determinarão se os trabalhadores serão marginalizados ou capacitados e se suas organizações estarão focadas mais na criação de valor para a sociedade ou na redução de custos. Não há um conjunto único de escolhas corretas. Mas quando os líderes do governo avaliam as tecnologias de IA, eles devem considerar quais opções maximizarão o valor público para os contribuintes e a sociedade.

Aos gestores públicos de recursos humanos, o cenário futuro revela grandes desafios. As tarefas dos líderes governamentais envolverão a reconfiguração das estruturas das carreiras públicas, tornando-as mais flexíveis e adaptáveis às mudanças ecossistêmicas e tecnológicas no mercado de trabalho. Eles também devem promover a educação para requalificar os servidores públicos frente às mudanças e aos desafios decorrentes do uso crescente de tecnologias emergentes para automação no setor público.

Também será necessário provocar inovações no ambiente de trabalho com vista a possibilitar aos trabalhadores migrarem para um novo paradigma no qual as inter-relações entre pessoas e entre humanos-máquina, bem como a criatividade serão mais importantes que processos $e$ rotinas. Será importante criar programas inovadores de trabalho remoto e semirremoto, com flexibilização de horários e locais de trabalho. Restará aos governos os devidos ajustes entre a força de trabalho humana e a IA, buscando equilibrar os ganhos evidentes da adoção dessas tecnologias no setor público com os seus possíveis efeitos na força de trabalho humana.

Por fim, a pesquisa bibliográfica apresentada neste trabalho limitou-se à base de dados Scopus, enquanto o levantamento patentário limitou-se à base Questel Orbit, pois essas bases de dados se caracterizam como relevantes de resumos e citações de literatura científica, bem como de documentos de patentes, respectivamente. Portanto, o tema precisa ser estudado com maior profundidade e abrangência contemplando demais bases igualmente importantes, o que fica como sugestão para trabalhos futuros.

\section{Referências}

ADAMS, S. et al. Mapping the Landscape of Human-Level Artificial General Intelligence. AI Magazine, [s.l.], v. 33, n. 1, p. 25-42, 15 mar. 2012.

ANDROUTSOPOULOU, A. et al. Transforming the communication between citizens and government through AI-guided chatbots. Government Information Quarterly, [s.l.], v. 36, n. 2, p. 358-367, abr. 2019.

ANSIP, A. Making the Most of Robotics and Artificial Intelligence in Europe. [2017].

Disponível em: https:/ec.europa.eu/commission/commissioners/2014-2019/ansip/blog/making-mostrobotics-and-artificial-intelligence-europe_en. Acesso em: 4 jun. 2019. 
BASTRA, G.; QUEIROLO, A.; SANTHANAM, N. Artificial intelligence: the time to act is now. Institucional. [2018]. Disponível em: https://www.mckinsey.com/industries/advanced-electronics/ourinsights/artificial-intelligence-the-time-to-act-is-now. Acesso em: 31 maio 2019.

BHATTACHARYYA, S. S.; NAIR, S. Explicating the future of work: perspectives from India. Journal of Management Development, [s.l.], v. 38, n. 3, p. 175-194, 8 abr. 2019.

BORRY, E. L.; GETHA-TAYLOR, H. Automation in the Public Sector: Efficiency at the Expense of Equity? Public Integrity, [s.l.], v. 21, n. 1, p. 6-21, 19 mar. 2018.

BROWN, J. et al. Workforce of the future: the competing forces shaping 2030. [S.l]: PwC, 20 jun. 2018.

BOYD, M.; WILSON, N. Rapid developments in Artificial Intelligence: how might the New Zealand government respond? Policy Quarterly, [s.l.], v. 13, n. 4, $1^{\circ}$ nov. 2017.

CAVALCANTE, P.; CAMÕES, M. Public innovation in Brazil: an overview of its types, results and drivers. [2017]. Disponível em: http://www.ipea.gov.br. Acesso em: 2 abr. 2017.

CENTRE FOR PUBLIC IMPACT. Destination Unknown AI and government. [S.I.]:

BCG Foundation, set. 2017. Disponível em: https://publicimpact.blob.core.windows.net/ production/2017/09/Destination-Unknown-AI-and-government.pdf. Acesso em: 2 jul. 2019.

CHATFIELD, A. T.; REDDICK, C. G. A framework for Internet of Things-enabled smart government: A case of IoT cybersecurity policies and use cases in U.S. federal government. Government Information Quarterly, [s.l.], v. 36, n. 2, p. 1-10, 18 set. 2018.

CHINA Issues Guideline on Artificial Intelligence Development. [2017]. Disponível em: http://english. gov.cn/policies/latest_releases/2017/07/20/content_281475742458322.htm. Acesso em: 4 jun. 2019.

COLLIER, M. et al. Artificial Intelligence: Healthcare's New Nervous System. [S.l.]: Accenture, 2017. Acesso em: 3 jul. 2019.

DELOITTE. Artificial Intelligence Innovation Report. [S.l.]: Deloitte, 2018. Disponível em: https:/www2.deloitte.com/content/dam/Deloitte/de/Documents/Innovation/Artificial-IntelligenceInnovation-Report-2018-Deloitte.pdf. Acesso em: 4 jun. 2019.

DESOUZA, K. C. Delivering Artificial Intelligence in Government: Challenges and Opportunities. Washington, DC: IBM Center for the Business of Government, 2018. Disponível em: http://www.businessofgovernment.org/sites/default/files/Delivering\%20Artificial\%20Intelligence\%20 in\%20Government.pdf. Acesso em: $1^{\circ}$ jul. 2019.

FREY, C. B.; OSBORNE, M. A. The future of employment: How susceptible are jobs to computerisation? Technological Forecasting and Social Change, [s.l.], v. 114, 17 set. 2013.

FUTURE TODAY INSTITUTE. Tech Trends Report 2019: Emerging science and technology trends that will influence business, government, education, media and society in the coming year. [S.l.] Future Today Institute, 2019. Disponível em: https://futuretodayinstitute.com/2019-tech-trends/. Acesso em: 5 jun. 2019.

GOLDSMITH, S.; CRAWFORD, S. The Responsive City: Engaging Communities Through DataSmart Governance. [S.l.] John Wiley \& Sons, 2014.

HOLDREN, J. P.; SMITH, M. Preparing for the Future of Artificial Intelligence. National Science and Technology Council, 12 out. 2016. Disponível em: https://obamawhitehouse.archives.gov/sites/default/files/ whitehouse_files/microsites/ostp/NSTC/preparing_for_the_future_of_ai.pdf. Acesso em: 4 jun. 2019. 
KANKANHALLI, A.; CHARALABIDIS, Y.; MELLOULI, S. IoT and AI for Smart Government: a Research Agenda. Government Information Quarterly, [s.l.], v. 36, n. 2, p. 304-309, abr. 2019.

KOUZIOKAS, G. N. The application of artificial intelligence in public administration for forecasting high crime risk transportation areas in urban environment. Transportation Research Procedia, 3rd Conference on Sustainable Urban Mobility, 3rd CSUM 2016, 26 - 27 May 2016, Volos, Greece. v. 24, p. 467-473, $1^{\circ}$ jan. 2017.

LEGG, S.; HUTTER, M. A Collection of Definitions of Intelligence. Frontiers in Artificial Intelligence and Applications, [s.l.], v. 157, p. 9, 25 jun. 2007.

MANYIKA, J. et al. A future that works: Automation, employment, and productivity. [S.l.] McKinsey Global Instituto, jan. 2017. Disponível em: https://www.mckinsey.com/ /media/McKinsey/ Featured\%20Insights/Digital\%20Disruption/Harnessing\%20automation\%20for\%20a\%20future\%20 that\%20works/MGI-A-future-that-works_Full-report.ashx. Acesso em: 5 jun. 2019.

MARCONI, M. de A.; LAKATOS, E. M. Fundamentos de Metodologia Científica. 5. ed. São Paulo: Atlas, 2003.

MARTINS, G. de A. Manual para Elaboração de Monografias e Dissertações. 2. ed. São Paulo: Atlas, 2000.

MCCARTHY, J. et al. A Proposal for the Dartmouth Summer Research Project on Artificial Intelligence. AI Magazine, [s.l.], v. 27, n. 4, p. 12-12, 15 dez. 2006.

MEHR, H. Artificial Intelligence for Citizen Services and Government. Harvard Ash Center Technology \& Democracy Fellow, [s.l.], p. 19, 2017.

MIKHAIL, B.; ALEKSEI, M.; EKATERINA, S. On the Way to Legal Framework for AI in Public Sector. Proceedings of the 11th International Conference on Theory and Practice of Electronic, 2018.

NILSSON, N. J. The Quest for Artificial Intelligence: a History of Ideas and Achievements. Cambridge, UK: Cambridge University Press, 2010.

PATIL, A. P. et al. Applying Machine Learning Techniques for Sentiment Analysis in the Case Study of Indian Politics. (S. M. Thampi et al. ed.). In: ADVANCES IN SIGNAL PROCESSING AND INTELLIGENT RECOGNITION SYSTEMS. Advances in Intelligent Systems and Computing. Springer International Publishing, 2018. Anais [...]. 2018.

QUESTEL. Orbit Intelligence. [2019]. Disponível em: https://www.questel.com/businessintelligence-software/orbit-intelligence/. Acesso em: 20 abr. 2021.

REIS, J.; SANTO, P. E.; MELÃO, N. Artificial Intelligence in Government Services: A Systematic Literature Review. (Á. Rocha et al. ed.). In: NEW KNOWLEDGE IN INFORMATION SYSTEMS AND TECHNOLOGIES. Anais [...]. Advances in Intelligent Systems and Computing. Springer International Publishing, 2019.

RICH, E.; KNIGHT, K.; NAIR, S. B. Artificial Intelligence. New Dehli; India: Third Edition ed.; McGraw-Hill Education, 2009.

ROSA, M.; FEYEREISL, J.; COLLECTIVE, T. G. A Framework for Searching for General Artificial Intelligence. Cornell University, [s.l.], 2 nov. 2016. 
SMITH, A. Future of Workforce Automation: America's Predictions. Pew Research Center, [s.l.], 10 mar. 2016.

STONE, P. et al. Artificial Intelligence and Life in $\mathbf{2 0 3 0}$ - One hundred year study on artificial intelligence - Report of the 2015-2016 Study Panel: Artificial Intelligence and Life in 2030. Stanford, CA: Stanford University, 2016. Disponível em: http://ai100.stanford.edu/2016-report. Acesso em: 31 maio 2019.

SUN, T. Q.; MEDAGLIA, R. Mapping the challenges of Artificial Intelligence in the public sector: Evidence from public healthcare. Government Information Quarterly, [s.l.], v. 36, n. 2, p. 368383, abr. 2019.

THIERER, A.; O'SULLIVAN, A. C.; RUSSELL, R. Artificial Intelligence and Public Policy. Mercatus Research, [s.l.], p. 56, 2017.

WELLER, C. Bill Gates says robots that take your job should pay taxes. [2017]. Disponível em: https://www.businessinsider.com/bill-gates-robots-pay-taxes-2017-2. Acesso em: 26 jun. 2019.

WIRTZ, B. W.; WEYERER, J. C.; GEYER, C. Artificial Intelligence and the Public Sector Applications and Challenges. International Journal of Public Administration, [s.l.], v. 42, n. 7, p. 1-13, 19 maio 2019.

\section{Sobre os Autores}

\section{Washington Fernando da Silva}

E-mail: meuconsultorfunciona@gmail.com

ORCID: http://orcid.org/0000-0002-1665-5971

Mestre em Propriedade Intelectual e Transferência de Tecnologia para Inovação (área Propriedade Intelectual) pelo PROFNIT-UFMT em 2021.

Endereço profissional: Governo do Estado de Mato Grosso, Centro Político Administrativo, s/n, Bloco III, Complexo Paiaguás, Rua C, Cuiabá, MT. CEP: 78049-005.

\section{Fernando Selleri Silva}

E-mail: selleri@unemat.br

ORCID: http://orcid.org/0000-0003-2158-4028

Doutor em Ciência da Computação pelo Centro de Informática (CIn) da Universidade Federal de Pernambuco em 2015.

Endereço profissional: Faculdade de Ciências Exatas e Tecnológicas, Universidade do Estado de Mato Grosso (UNEMAT), Caixa Postal 92, Barra do Bugres, MT. CEP: 78390-000.

\section{Olivan da Silva Rabêlo}

E-mail: olivanrabelo@gmail.com

ORCID: http://orcid.org/0000-0002-4940-8440

Doutor em Economia Aplicada pela Universidade Federal de Pernambuco (UFPE) em 2015.

Endereço profissional: Universidade Federal de Mato Grosso, Av. Fernando Corrêa da Costa, n. 2.367, Bairro Boa Esperança, Cuiabá, MT. CEP: 78060-900. 